\title{
Reproductive Endocrinology
}

\author{
Lena Sahlin and Olle Söder \\ Paediatric Endocrinology Unit, Department of Women's and Children's Health, Astrid Lindgren Children's \\ Hospital, Karolinska Institutet and Karolinska University Hospital, Stockholm, Sweden
}

\begin{abstract}
This chapter has a selection of papers published during the last year dealing with many important aspects of reproductive endocrinology. They include endocrine treatments, gender differences in brain imaging, care for children with gender dysphoria, genetic studies in disorders of sexual development and reproductive disorders, puberty, markers of gonadal function and many others. The chapter aims at presenting a mix of experimental and clinical papers advancing the field of reproductive endocrinology with implications for a better understanding of human physiology and better care for children with endocrine disorders. The present selection of papers obviously represents our own bias but we hope you find them interesting to read and helpful for your daily professional activity. There is limited space for everything to be covered within the frame of this chapter but there are also additional publications dealing with subjects related to reproductive endocrinology in other chapters of this Yearbook.
\end{abstract}

\section{New treatments}

\section{Aromatase inhibitors in pediatrics}

Wit JM, Hero M, Nunez SB

Department of Pediatrics, J6S, Leiden University Medical Center, Leiden, The Netherlands

j.m.wit@lumc.nl

Nat Rev Endocrinol 2012;8:135-147

Background: The enzyme CYP19 aromatase catalyzes the rate-limiting step in the conversion of androgens to estrogens in many tissues. Translational studies have revealed a major role for this enzyme in epiphyseal plate closure, which has promoted interest in the use of inhibitors of aromatase to improve longitudinal growth and adult height of children. The relatively novel selective aromatase inhibitors letrozole and anastrozole have been reported to be used off-label in pediatrics for several medical conditions.

Methods: This paper reviews the reported use of aromatase inhibitors in pediatric practice. Their use in the following medical conditions in children is presented: hyperestrogenism, such as aromatase excess syndrome, Peutz-Jeghers syndrome, McCune-Albright syndrome and functional follicular ovarian cysts; hyperandrogenism, e.g. testotoxicosis (also known as familial male-limited precocious puberty), congenital adrenal hyperplasia; pubertal gynecomastia, and short stature and/or pubertal delay in boys.

Results: Reported data suggest that aromatase inhibitors are probably effective in the treatment of patients with aromatase excess syndrome or testotoxicosis, partially effective in Peutz-Jeghers and McCune-Albright syndrome, but probably ineffective in gynecomastia. Insufficient data are available in patients with congenital adrenal hyperplasia or functional ovarian cysts.

Conclusions: Aromatase inhibitors appear effective in increasing adult height of boys with short stature and/or pubertal delay but safety concerns, including vertebral deformities, a decrease in serum HDL cholesterol levels and increase of erythrocytosis, are reasons for caution.

Aromatase inhibitors seem to have a role in the treatment of certain rare endocrine disorders in children but these patients should be handled by specialists with an ambition to add an accumulating experience to increase the evidence base. Additional studies with long-lasting follow-up are required to understand better the efficacy and safety of these drugs in pediatric endocrinology. At the present stage of knowledge, common use of these potent drugs to improve longitudinal growth of boys is not recommended. 


\section{Effect of oxandrolone and timing of pubertal induction on final height in Turner's syndrome: randomized, double-blind, placebo-controlled trial}

Gault EJ, Perry RJ, Cole TJ, Casey S, Paterson WF, Hindmarsh PC, Betts P, Dunger DB, Donaldson MD

University of Glasgow Department of Child Health, Royal Hospital for Sick Children, Glasgow, UK

BMJ 2011;342:d1980

Background: The nonaromatizable androgen oxandrolone has been used as an adjuvant to stimulate longitudinal growth in children including those with Turner's syndrome. This clinical study examines the effect of oxandrolone and the timing of pubertal induction on final height in girls with Turner's syndrome receiving a standard dose of growth hormone.

Methods: A randomized, double-blind, placebo-controlled trial was performed in the settings of 36 pediatric endocrinology departments in UK hospitals. Girls with Turner's syndrome aged 7-13 years at recruitment, receiving recombinant growth hormone therapy $\left(10 \mathrm{mg} / \mathrm{m}^{2} / \mathrm{week}\right)$ were included. Participants were randomized to oxandrolone $(0.05 \mathrm{mg} / \mathrm{kg} /$ day, maximum $2.5 \mathrm{mg} /$ day $)$ or placebo from 9 years of age. Those with evidence of ovarian failure at 12 years were further randomized to oral ethinylestradiol in a controlled dose schedule or placebo; participants who received placebo and those recruited after the age of 12.25 years started ethinylestradiol at age 14 . Final height was the main outcome measure.

Results: 106 participants were recruited, of whom 14 withdrew and 82/92 reached final height. Both oxandrolone and late pubertal induction increased final height: by 4.6 (95\% confidence interval $1.9-$ $7.2) \mathrm{cm}(\mathrm{p}=0.001, \mathrm{n}=82)$ for oxandrolone and $3.8(0.0-7.5) \mathrm{cm}(\mathrm{p}=0.05, \mathrm{n}=48)$ for late pubertal induction with ethinylestradiol. In the 48 children who were randomized twice, the effects on final height (compared with placebo and early induction of puberty) of oxandrolone alone, late induction alone, and oxandrolone plus late induction were similar, averaging $7.1(3.4-10.8) \mathrm{cm}(\mathrm{p}<0.001)$. No cases of virilization were reported.

Conclusion: Oxandrolone had a positive effect on final height in girls with Turner's syndrome treated with growth hormone, as did late pubertal induction with ethinylestradiol at age 14 years. However, these effects were not additive, so using both had no advantage. Oxandrolone could, therefore, be offered as an alternative to late pubertal induction for increasing final height in Turner's syndrome.

This study shows convincingly that oxandrolone promotes longitudinal growth in girls with Turner's syndrome treated with growth hormone. Delaying puberty by later start of estrogen therapy in these patients treated with oxandrolone had no further effect on their final height. Oxandrolone thus allows an early start of pubertal induction without compromising final height. This is good news since many young adult Turner patients report they did suffer more from the delayed puberty in their teen age rather than the loss of a few centimeters in final height.

\section{New genes (and old)}

\section{Etiology of hypospadias: a systematic review of genes and environment}

Van der Zanden LF, van Rooij IA, Feitz WF, Franke B, Knoers NV, Roeleveld N

Department of Epidemiology, Biostatistics and HTA, Radboud University Nijmegen Medical Centre, Nijmegen,

The Netherlands

Hum Reprod Update 2012;18:260-283

Background: Hypospadias is a common congenital malformation of the male external genitalia and a sign of undermasculinization during prenatal development. Most cases have an unknown etiology, which is probably a mix of monogenic and multifactorial forms, implicating both genes and environmental factors.

Method: The authors reviewed the current knowledge about the etiology of hypospadias. PubMed was used to identify studies on hypospadias etiology published between January 1995 and February 2011. Reference lists of the selected articles were also searched to identify additional studies, including those published before 1995 . 
Results: The search provided 922 articles and 169 were selected for this review. Studies screening groups of patients with hypospadias for single gene defects found mutations in WT1, SF1, BMP4, BMP7, HOXA4, HOXB6, FGF8, FGFR2, AR, HSD3B2, SRD5A2, ATF3, MAMLD1, MID1 and BNC2. However, most investigators were convinced that single mutations do not cause the majority of isolated hypospadias cases. Indeed, associations were found with common polymorphisms in FGF8, FGFR2, AR, HSD17B3, SRD5A2, ESR1, ESR2, ATF3, MAMLD1, DGKK, MID1, CYP1A1, GSTM1 and GSTT1. In addition, gene expression studies identified CTGF, CYR61 and EGF as candidate genes. Environmental factors consistently implicated in hypospadias were low birth weight, maternal hypertension and preeclampsia, suggesting that placental insufficiency may play an important role in hypospadias etiology. Exogenous endocrine-disrupting chemicals have the potential to induce hypospadias, but it is unclear whether human exposure is high enough to exert this effect. Other environmental factors have also been associated with hypospadias but, for most, the results are inconsistent.

Conclusions: A number of contributors to the etiology of hypospadias have been identified, but the majority of risk factors still remain unknown.

Despite its common nature and results of multiple extensive studies searching for causes, the etiology of hypospadias still remains an enigma in most cases. Due to poor reporting it has been difficult to find hard evidence that the incidence of hypospadias is increasing although some studies point to such trends, at least in certain geographical regions. This may implicate a role for environmental factors in the pathogenesis and most focus has been on endocrine-disrupting chemicals with antiandrogenic actions, hypothetically operating at critical time windows of male embryonic and fetal development. More studies looking for causative links of hypospadias are highly warranted.

\section{FOXL2 impairment in human disease}

Verdin $\mathrm{H}$, De Baere $\mathrm{E}$

Center for Medical Genetics, Ghent University Hospital, Ghent, Belgium

Horm Res Paediatr 2012;77:2-11

\section{Missense mutation outside the forkhead domain of FOXL2 causes a severe form of BPES type II}

Haghighi A, Verdin H, Haghighi-Kakhki H, Piri N, Gohari NS, Baere ED

Wellcome Trust Centre for Human Genetics, University of Oxford, Oxford, UK

Mol Vis 2012;18:211-218

Background: FOXL2 encodes a forkhead transcription factor that plays important roles in the ovary during development and in postnatal, adult life. FOXL2 impairment will, in line with other forkhead transcription factors, result in constitutional genetic defects and a somatic mutation which will lead to developmental disease and cancer, respectively. More than 100 unique constitutional mutations and regulatory defects have been found in blepharophimosis syndrome (BPES), a complex eyelid malformation associated (type I) or not (type II) with premature ovarian failure (POF). In agreement with the BPES phenotype, FOXL2 is expressed in the developing eyelids and in fetal and adult ovaries.

Methods: Article 1: Review of known models and patients with FOXL impairment. Article 2: A clinical and molecular genetic investigation was performed in affected and unaffected members of an Iranian family with BPES. The FOXL2 coding region was sequenced in an index case. Targeted mutation testing was performed in 8 family members.

Results: Article 1: Only a few constitutional mutations have been described in nonsyndromic POF. A recurrent somatic mutation p.C134W was found to be specific for adult ovarian granulosa cell tumors. A conditional knockout of Foxl2 in the mouse induced somatic transdifferentiation of ovary into testis in adult mice, suggesting that Foxl2 has an anti-testis function in the adult ovary. Article 2: A heterozygous FOXL2 missense mutation c.650C $\rightarrow G$ (p.Ser217Cys) cosegregating with disease in members of a three-generation family with BPES type II was identified. Only few missense mutations have been reported outside the forkhead domain so far, which were all found in mild BPES. Unlike previous studies, affected members of the family studied here showed a severe BPES phenotype, with bilateral amblyopia due to uncorrected ptosis. 
Conclusions: The transdifferentiation of the ovary into a testis has changed the view of the gonads as terminally differentiated organs in adult mammals. This result might have potential implications for the understanding and treatment of conditions such as POF and polycystic ovary syndrome.

A severe BPES phenotype resulting from a FOXL2 missense mutation outside the forkhead domain was demonstrated, expanding knowledge of the phenotypic consequences of missense mutations outside the forkhead domain.

These two articles present FOXL2 as an important gene for which an impairment has important clinical consequences resulting in human disease. Not all BPES syndromes are accompanied by POF in female patients, although the gene is expressed in the eyelids and ovarian tissues. It is clear that FOXL2 has an important function for differentiation of the gonads.

\section{Important for clinical practice}

\section{The physiology and timing of male puberty}

Tinggaard J, Mieritz MG, Sorensen K, Mouritsen A, Hagen CP, Aksglaede L, Wohlfahrt-Veje C, Juul A

Department of Growth and Reproduction, Rigshospitalet, Faculty of Health Sciences, University of Copenhagen,

Copenhagen, Denmark

Curr Opin Endocrinol Diabetes Obes 2012;19:197-203

Background: An earlier start of puberty during the past few decades been implicated and associated with environmental factors such as the obesity epidemic. However, the evidence base for such secular trends and environmental associations is greater in girls than in boys and more studies on gender differences are therefore warranted.

Methods: The authors aimed to describe available markers of male puberty, discuss associations between adiposity and pubertal timing and to review recent evidence of a possible secular trend in male pubertal timing.

Results: An expert panel reviewed existing American pubertal data from boys in 2005 and could not confirm a secular trend in male pubertal timing. National Health and Nutrition Examination Survey III findings have been confirmed by the National Institute of Child Health and Human Development study reporting a mean age of 10.4 years for Caucasian boys entering Tanner stage G2. Furthermore, the Copenhagen Puberty Study reported a 3-month decline in pubertal onset during a 15-year period (from 11.92 years in 1991 to 11.66 years in 2008). A negative association between obesity and early puberty was found in the National Institute of Child Health and Human Development study, in contrast to the positive association found in a Danish study. Other studies have not been able to document an association between prepubertal BMI and age at pubertal onset.

Conclusions: Evaluation of Tanner stage and especially assessment of testicular volume should both be used in epidemiological studies. The authors speculate that the association between fat mass and pubertal timing may be nonlinear and recent studies may indicate a small decline in age at pubertal onset in boys.

This review addresses the gender difference in the recently observed secular trend to earlier onset of puberty. Most studies have investigated this phenomenon in girls but not in boys, and studies addressing boys have conflicting results. This difference may have a methodological explanation due to the greater difficulties to examine boys than girls for such studies. However a difference in biology between boys and girls with respect to onset of puberty cannot be neglected, also displayed by the well-known gender differences in the incidences of precocious and delayed puberty. Speculatively, from a biological point of view, it makes more sense that overnutrition (with or without obesity) in boys results in delayed puberty reflecting less physical fitness in obese men whereas obese women may be nutritionally fit for reproduction. 


\section{Sertoli cell markers in the diagnosis of pediatric male hypogonadism}

Grinspon RP, Loreti N, Braslavsky D, Bedecarras P, Ambao V, Gottlieb S, Bergada I, Campo SM, Rey RA

Centro de Investigaciones Endocrinológicas (CEDIE, CONICET), Hospital de Niños Ricardo Gutiérrez, Buenos Aires,

Argentina

J Pediatr Endocrinol 2012;25:3-11

Background: During childhood, the pituitary-testicular axis is partially dormant: testosterone secretion decreases following a drop in luteinizing hormone (LH) levels; follicle-stimulating hormone (FSH) levels also go down. Conversely, Sertoli cells are most active, as revealed by the circulating levels of antimüllerian hormone $(\mathrm{AMH})$ and inhibin $\mathrm{B}$. Therefore, hypogonadism can best be evidenced, without stimulation tests, if Sertoli cell function is assessed.

Methods: This paper reviews markers of testicular function in children and discusses their clinical implications.

Results: Serum AMH levels in boys are high from fetal life until midpuberty. Testicular AMH production increases in response to FSH and is potently inhibited by androgens. Inhibin B is high in the first years of life, then decreases partially while remaining clearly higher than in females, and increases again at puberty. Serum AMH and inhibin B are undetectable in anorchid patients. In primary or central hypogonadism affecting the whole gonad established in fetal life or childhood, all testicular markers are low. Conversely, when hypogonadism only affects Leydig cells, serum AMH and inhibin B are normal. In males of pubertal age with central hypogonadism, AMH and inhibin B are low. Treatment with FSH provokes an increase in serum levels of both Sertoli cell markers, whereas human chorionic gonadotrophin (hCG) administration increases testosterone levels.

Conclusions: Measurements of serum AMH and inhibin B are helpful in assessing testicular function, without need for stimulation tests, and orientates the etiological diagnosis of pediatric male hypogonadism.

This review is a helpful summary of circulating biomarkers of testicular function to be used in clinical practice. AMH and inhibin B are markers of Sertoli cell function, which show age- and maturational stage-specific patterns in blood samples. By combining these factors with classical endocrine tests (gonadotropins) and Leydig cell markers (testosterone, INSL3) it is possible to get a good overview of testicular function without performing more cumbersome investigations including endocrine stimulation tests. See also the paper below by Dennis et al. in this chapter, discussing the role of vitamin D in $\mathrm{AMH}$ production.

\section{Children and adolescents with gender identity disorder referred to a pediatric medical center}

Spack NP, Edwards-Leeper L, Feldman HA, Leibowitz S, Mandel F, Diamond DA, Vance SR

Division of Endocrinology, Children's Hospital Boston, Boston, MA, USA

norman.spack@childrens.harvard.edu

Pediatrics 2012;129:418-425

Background: An increasing number of children and adolescents are seeking medical and psychological help for gender dysphoria. This paper aims at describing patients with gender identity disorder (GID) referred to a pediatric medical center. The authors identified changes in patient profiles and numbers after creation of a multidisciplinary gender management service by expanding the disorders of sex development clinic to include transgender patients.

Methods: Data were collected on 97 consecutive patients aged $<21$ years, with initial visits between January 1998 and February 2010, who fulfilled the following criteria: long-standing cross-gender behaviors, provided letters from current mental health professional and parental support. Main descriptive measures included gender, age, Tanner stage, history of gender identity development, and psychiatric comorbidity.

Results: Genotypic male:female ratio was 43:54 (0.8:1); there was a slight preponderance of female patients but not significantly different from $1: 1$. Age of presentation was $14.8 \pm 3.4$ years $($ mean \pm SD) without sex difference $(\mathrm{p}=0.11)$. Tanner stage at presentation was $4.1 \pm 1.4$ for genotypic female patients and $3.6 \pm 1.5$ for genotypic male patients $(\mathrm{p}=0.02)$. Age at start of medical treatment was 15.6 \pm 2.8 years. 43 patients $(44 \%)$ presented with significant psychiatric history, including 20 reporting self-mutilation $(21 \%)$ and suicide attempts $(9 \%)$. 
Conclusions: After establishment of a multidisciplinary gender clinic, the GID population increased 4-fold. Complex clinical presentations required additional mental health support as the patient population grew. Mean age and Tanner stage were too advanced for pubertal suppressive therapy to be an effective option for most patients. Two-thirds of patients were started on cross-sex hormone therapy. Greater awareness of the benefit of early medical intervention is needed. Psychological and physical effects of pubertal suppression and/or cross-sex hormones in our patients require further investigation.

Young patients with GID often report they are misunderstood or neglected by the healthcare system. These patients often show various forms of comorbidity and behavioral disturbances including self-destructive activities and drug abuse that may be related to the lack of adequate care. It is therefore important to recognize this condition and offer psychiatric and endocrine expertise. This paper describes a dramatic increase in the number of GID patients after opening a specific clinic for such problems. The gender balance of these young patients shows more female to male rather than male to female transgenderism which is opposite to that reported for adult patients. The reason for this is unknown. The role of the pediatric endocrinologist for these patients is to be responsible for the work-up to reveal 'cryptic' disorders of sexual development, e.g. $5 \alpha$-reductase deficiency, and to care for the endocrine treatment before adult age has been reached.

\section{New mechanisms}

\section{Targeted pituitary overexpression of pituitary adenylate-cyclase activating polypeptide alters postnatal sexual maturation in male mice}

Moore JP Jr, Yang RQ, Winters SJ

Department of Anatomical Sciences and Neurobiology, University of Louisville, School of Medicine, Louisville, KY, USA

jpmoor03@louisville.edu

Endocrinology 2012;153:1421-1434

Background: The mechanisms behind pubertal onset and its molecular background are yet to be fully understood although many downstream signals and pathways have been discovered. The neuropeptide pituitary adenylate cyclase-activating polypeptide (PACAP) is present in high concentrations within the hypothalamus, suggesting a trophic role to the pituitary, whereas pituitary expression suggests a paracrine function. PACAP is known to stimulate gonadotropin secretion and enhances GnRH responsiveness. PACAP increases gonadotropin $\alpha$-subunit ( $\alpha$-GSU), lengthens LH- $\beta$, but reduces FSH- $\beta$ mRNA levels in adult pituitary cell cultures in part by increasing follistatin. PACAP stimulates LH secretion in rats, however the acceptance of PACAP as a regulator of reproduction has been limited by lack of results from in vivo studies.

Methods: The authors created a transgenic mouse model of pituitary PACAP overexpression using the $\alpha$-GSU subunit promoter. Real-time PCR was used to evaluate PACAP, follistatin, GnRH receptor, and the gonadotropin subunit mRNA in male transgenic and wild-type mice of various ages.

Results: Transgenic mice had greater than 1,000-fold higher levels of pituitary PACAP mRNA, and immunocytochemistry, Western blot, and ELISA analyses confirmed high peptide levels. FSH, LH, and testosterone levels were significantly suppressed, and the timing of puberty was substantially delayed in PACAP transgenic mice in which gonadotropin subunit and GnRH receptor mRNA levels were reduced and pituitary follistatin expression was increased. Microarray analyses revealed 1,229 of 45,102 RNA probes were significantly $(\mathrm{p}<0.01)$ different in pituitaries from PACAP transgenic mice, of which 83 genes were at least 2-fold different. Genes involved in small molecule biochemistry, cancer, and reproductive system diseases were the top associated networks. The GnRH signaling pathway was the top canonical pathway affected by pituitary PACAP excess.

Conclusions: These results provide the first evidence that PACAP affects gonadotropin expression and sexual maturation in vivo. Clinical implications of these findings await further studies. 
PACAP is yet another factor that may be involved in the regulation of time of onset and tempo of puberty. This peptide seems to suppress the start and halt the progression of pubertal development although its clinical role is yet to be defined. Its action seems to be more paracrine than endocrine, so unfortunately it is difficult to believe that circulating PACAP levels could be informative with regard to pubertal timing. However, a pharmacological role of PACAP in disorders of puberty may be worth exploring.

\section{The level of serum anti-müllerian hormone correlates with vitamin D status in men and women but not in boys}

Dennis NA, Houghton LA, Jones GT, van Rij AM, Morgan K, McLennan IS

Department of Anatomy (N.A.D., K.M., I.S.M.), Otago School of Medical Sciences and Departments of Human

Nutrition (L.A.H.), Psychology (K.M.), and Surgery (G.T.J., A.M.v.R.), Dunedin School of Medicine, and Brain Health

Research Centre (N.A.D., I.S.M.), University of Otago, Dunedin, New Zealand

J Clin Endocrinol Metab 2012;97:2450-2455

Background: Anti-müllerian hormone $(\mathrm{AMH})$ is a gonad-specific hormone, which is increasingly used as a marker of gonadal status. The level of serum AMH has a high variance in similar individuals for reasons that are unknown. The AMH gene promoter contains a vitamin D response element that may cause vitamin D status to influence serum AMH levels. The objective of this study was to determine whether serum levels of $\mathrm{AMH}$ are related to 25-hydroxyvitamin $\mathrm{D}([25(\mathrm{OH}) \mathrm{D})]$ status.

Methods: Three cohorts of participants were analyzed in this observational and intervention study. The cohorts were mature men $(\mathrm{n}=113)$, premenopausal women $(\mathrm{n}=33)$, and 5 - to 6 -year-old boys $(\mathrm{n}=74)$. Women were given a daily supplement of ergocalciferol, cholecalciferol, or a placebo for 6 months and provided baseline and posttreatment blood samples. Serum AMH and 25(OH)D were measured and analyzed for covariation.

Results: Serum AMH positively correlated with 25(OH)D in men $(r=0.22, \mathrm{p}=0.02)$ but not boys. Both $25(\mathrm{OH}) \mathrm{D}$ and $\mathrm{AMH}$ levels exhibited seasonal variation in women, with an $18 \%$ decrease in $\mathrm{AMH}$ levels in winter compared with summer $(\mathrm{p}=0.01)$. Change in $\mathrm{AMH}$ level correlated with the initial $\mathrm{AMH}$ level and the magnitude of change in vitamin D levels $(r=0.36, \mathrm{p}=0.004)$. Cholecalciferol supplementation prevented seasonal AMH change.

Conclusion: Vitamin D may be a positive regulator of AMH production in adults, and vitamin D deficiency may confound clinical decisions based on AMH. Vitamin D status should be considered when serum AMH levels are obtained for diagnosis.

This paper demonstrates that vitamin D amongst many other functions is also involved in reproductive endocrinology, more specifically in regulation of $\mathrm{AMH}$ production. The message is that vitamin D status should be checked and any deficiency corrected before results of AMH analyses are interpreted. As the authors point out, the present findings may at least partially explain the great variability in AMH levels in different subjects with similar medical conditions. Further studies are required to understand better the specific physiological role of vitamin $\mathrm{D}$ in $\mathrm{AMH}$ regulation and gonadal function, if any, both prenatally and at different postnatal maturational stages.

\section{Ten novel mutations in the NR5A1 gene cause disordered sex development in $46, X Y$ and ovarian insufficiency in $46, X X$ individuals}

Camats N, Pandey AV, Fernandez-Cancio M, Andaluz P, Janner M, Toran N, Moreno F, Bereket A, Akcay T, Garcia-

Garcia E, Munoz MT, Gracia R, Nistal M, Castano L, Mullis PE, Carrascosa A, Audi L, Fluck CE

Pediatric Endocrinology (C.E.F.), Department of Pediatrics and Department of Clinical Research, University Children's

Hospital Bern, Bern, Switzerland

J Clin Endocrinol Metab 2012;97:E1294-E1306

Background: Steroidogenic factor-1 (SF-1/NR5A1) is a nuclear receptor that regulates adrenal and reproductive development and function. NR5A1 mutations have been detected in 46,XY individuals with disorders of sexual development (DSD) but apparently normal adrenal function and in 46, XX women with normal sexual development but yet primary ovarian insufficiency (POI). 
Methods: A group of 100 46,XY DSD and 2 POI patients were studied for NR5A1 mutations and their impact. Clinical, biochemical, histological, genetic, and functional characteristics of the patients with NR5A1 mutations are reported. Patients were referred from different centers in Spain, Switzerland, and Turkey. Histological and genetic studies were performed in Barcelona, Spain. In vitro studies were performed in Bern, Switzerland. A total of 65 Spanish and 35 Turkish patients with 46,XY DSD and 2 Swiss 46,XX patients with POI were investigated.

Results: Ten novel heterozygote NR5A1 mutations were detected and characterized (5 missense, 1 nonsense, 3 frameshift mutations, and 1 duplication). The novel NR5A1 mutations were tested in vitro by promoter transactivation assays showing grossly reduced activity for mutations in the DNA-binding domain and variably reduced activity for other mutations. The dominant negative effect of the mutations was excluded. The authors demonstrated high variability and thus no apparent genotype-structure-function-phenotype correlation. Histological studies of testes revealed vacuolization of Leydig cells due to fat accumulation.

Conclusions: SF-1/NR5A1 mutations are frequently found in 46,XY DSD individuals (9\%) and manifest with a broad phenotype. Testis histology is characteristic for fat accumulation and degeneration over time, similar to findings observed in patients with lipoid congenital adrenal hyperplasia (due to StAR mutations). Genotype-structure-function-phenotype correlation remains elusive.

The SF1/NR5A1 gene is an important regulator of prenatal sex differentiation affecting both adrenal and gonadal functions. An increasing number of mutations of this gene are being discovered and such involvement should be considered in cases of DSD with unknown background. In contrast to other well-studied genes with more defined functions, such as 21-hydroxylase in congenital adrenal hyperplasia, mutations of SF1/NR5A1 are much more difficult to interpret with respect to their functional consequences. Such lack of genotype-phenotype correlation also makes family counseling difficult and challenging.

\section{Food for thought}

\section{The science of sex - novel series in EMBO Reports}

Breithaupt $\mathrm{H}$ (ed)

EMBO Rep 2012;13:394

\section{Science \& Society Series on Sex and Science}

Sexual reproduction has facilitated the evolution of higher life forms and has had a profound influence on human history, culture and society. This series of invited papers in EMBO Reports explores the attempts to understand the influence of sex in the natural world, and the biological, medical and cultural aspects of sexual reproduction, gender and sexual pleasure.

\section{Sperm counts and fertility in men: a rocky road ahead}

Sharpe RM

MRC Centre for Reproductive Health, University of Edinburgh, The Queen's Medical Research Institute, Edinburgh, UK

EMBO Rep 2012;13:398-403

Synopsis of review: Both sperm counts and testosterone levels have been declining in men in recent decades which may suggest that male health might have also declined. There is a great incentive to understand what has caused lower sperm counts in men, and to establish whether this trend can be reversed or prevented. Falling sperm counts have been discussed as a scary story about environmental chemical pollution and unhealthy lifestyle changes but the evidence base for such conclusions is weak and the causes remain unknown. The fact that sperm counts have fallen across a short timescale of $50-70$ years is indeed a strong indication that the causes are related to lifestyle and environment, rather than genetic. 
This also means that the decline is probably preventable, and possibly reversible. For this to happen, the problem has to be recognized, its causes elucidated and appropriate intervention or prevention implemented. To identify the causes requires that one knows where and when to look. Sperm count probably matters more today than a few decades ago when most women had their children at an earlier age. But sperm count also matters for men for reasons even more fundamental than fertility. It is a sign of overall health; the lower your sperm count the greater your risk of dying. The other marker of healthy testis function - testosterone levels in blood - shows a similar relationship. Perhaps the most disturbing conclusion is that the evidence for low and falling sperm counts points to a wider issue of the subtle dysfunction of the process that makes men male. If this process is affected by maternal lifestyle and environmental exposures, which a growing body of evidence suggests is the case, then what other consequences for the programming of behavior and disease risks will it also bring?

This paper is an excellent review of the issue of decreasing sperm quality in Western societies during the past more than half a century. It brings up most of the relevant issues related to this important topic for critical discussion and points to important and necessary roads for future research. The paper is also the first in a series on Sex \& Science published in EMBO Reports, with a scientific approach to different aspects of sex that promises interesting reading. It is highly recommended for the pediatric endocrinologist who wants to remain updated on these important and interesting issues.

\section{Food for thought \\ Beware of the mattress!}

\section{Corncob bedding alters the effects of estrogens on aggressive behavior and reduces estrogen receptor- $\alpha$ expression in the brain}

Landeros RV, Morisseau C, Yoo HJ, Fu SH, Hammock BD, Trainor BC

Department of Psychology and Center for Neuroscience, University of California, Davis, Davis, CA, USA

Endocrinology 2012;153:949-953

Background: Estrogen signaling pathways can be modulated by naturally occurring environmental compounds such as phytoestrogens and xenoestrogens. Many researchers studying the effects of estrogens on brain function or behavior in animal models choose to use phytoestrogen-free food for this reason. Corncob bedding is commonly used in animal facilities across the United States and has been shown to inhibit estrogen-dependent reproductive behavior in rats. The mechanism for this effect was unclear, because the components of corncob bedding mediating this effect did not bind estrogen receptors.

Methods: The California mouse (Peromyscus californicus) was used and housed either on cardboard or corncob bedding.

Results: Estrogens decreased aggression when cardboard-based bedding was used, but this effect was absent when corncob bedding was used. California mice housed on corncob bedding also had fewer estrogen receptor- $\alpha$-positive cells in the bed nucleus of the stria terminalis and ventromedial hypothalamus compared with mice housed on cardboard-based bedding. In addition, corncob bedding suppressed the expression of phosphorylated ERK in these brain regions as well as in the medial amygdala and medial preoptic area.

Conclusion: The present observations on the effects of corncob bedding on behavior and brain function should draw attention to the importance that cage bedding can exert on neuroendocrine research.

This study lifts a warning flag worth noticing. A lot of effort has been put into reducing the impact from hormonal substances in food and cell medium to experimental animal and in vitro cultures. However, the cage bedding as well as the amusement toys for enrichment have not been thoroughly evaluated for unwanted hormonal side effects. The study by Landeros et al. is important to remind researchers that also cage inventories have to be cautiously chosen when doing studies where hormonal input might change the outcome. To pull these results a bit further and extrapolate to humans, one should be very careful when choosing bed material. Not many have mattresses stuffed with corncobs at home, but there are plenty of other materials which could very well contain, or be contami- 
nated with, phytoestrogens or xenoestrogens. Since we spend almost a third of our lives in bed, the exposure time for the material is very high. Modern mattresses usually contain stuffing such as latex, viscoelastic or other flexible polyurethane foams. Mattresses may also be filled with air or water, or a variety of natural fibers. Futons, the Japanese beds, are often stuffed with cotton, wool or synthetic batting. The pillows are filled with beans, buckwheat chaff or plastic beads. The plastics could contain estrogenic substances which could meddle with the neuroendocrine system, as could phytoestrogens, e.g. in beans. Next time you change your matrass - consider the options thoroughly!

\section{Food for thought}

The scientific evidence for the good outcome of a weekend trip!

\section{Short-term enrichment makes male rats more attractive, more defensive and alters hypothalamic neurons}

Mitra R, Sapolsky RM

School of Biological Sciences, Nanyang Technological University, Singapore, Singapore

PloS One 2012;7:e36092

Background: Innate behaviors are shaped by contingencies built during evolutionary history. On the other hand, environmental stimuli play a significant role in shaping behavior. Environmental enrichment is known to improve physiology and behavior at multiple levels in a variety of species. A short period of environmental enrichment (EE) can enhance cognitive behavior, modify effects of stress on learned behaviors and induce brain plasticity.

Methods: The authors investigated whether modulation by environment can extend to innate behaviors that are preserved by intense selection pressure, by studying effects of relatively short (14-day) environmental enrichment on two prominent innate behaviors in rats: avoidance of predator odors and ability of males to attract mates.

Results: Enrichment had strong effects on both the innate behaviors. The enriched males were more avoidant of a predator odor than nonenriched controls, and had a greater rise in corticosterone levels in response to the odor. The enriched males also had higher testosterone levels and were more attractive to females. A decrease in dendritic length of neurons of ventrolateral nucleus of hypothalamus was demonstrated. These neurons are important for reproductive mate choice and an increase of the same neurons in the dorsomedial nucleus is important also for defensive behavior. The possibility that these two sets of changes in neuronal cytoarchitecture are plausibly coupled (i.e. that increased predator avoidance is an adaptive compensation for the conspicuousness of increased sexual attraction) is exciting, and awaits further investigations.

Conclusions: Behavioral and hormonal observations provide evidence that a short period of EE can alter innate behaviors, providing a good example of gene-environment interaction.

What can we learn from this study? The EE was shown to have a strong impact on innate behaviors like attraction to females and avoidance of predators. If a female gives her partner a short-term enrichment, the result might be that he turns out to be more attractive to her. Thus, in the long run a recreational break like a weekend trip, could be what is needed for any long relationship to work out. The female will, besides enjoying the trip in itself, find the partner more attractive, although this might be a farfetched conclusion to draw from studies on rats. 


\section{Ovarian control of nectar collection in the honey bee (Apis mellifera)}

Siegel AJ, Freedman C, Page RE Jr

School of Life Sciences, Arizona State University, Tempe, AZ, USA

Plos One 2012;7:e33465

Background: Honey bees are a model system for the study of division of labor. Worker bees demonstrate a foraging division of labor (DOL) by biasing collection towards carbohydrates (nectar) or protein (pollen). There is a reproductive ground-plan hypothesis proposing that foraging DOL is regulated by the networks that controlled foraging behavior during the reproductive life cycle of honey bee ancestors.

Methods: A proposed mechanism was tested, through which the ovary of the facultatively sterile worker impacts foraging bias. The proposed mechanism suggests that the ovary has a regulatory effect on sucrose sensitivity, and sucrose sensitivity impacts nectar loading. This mechanism was tested by measuring worker ovary size (ovariole number), sucrose sensitivity, and sucrose solution load size collected from a rate-controlled artificial feeder. Three nonsimultaneous replicates were performed using 10 and $30 \%$ sucrose solutions.

Results: A significant interaction between ovariole number and sucrose sensitivity on sucrose solution load size was found when using low concentration nectar. This supports the proposed mechanism. As nectar and pollen loading are not independent, a mechanism impacting nectar load size would also impact pollen load size.

Conclusion: The results of this study demonstrate a link between ovariole number, sucrose sensitivity and nectar collection. These results support a proposed foraging DOL control mechanism where the ovary impacts sucrose responsiveness in honey bees. Sucrose responsiveness, in turn, impacts the loading of sugar-rich nectar. This mechanism fits well into the evolutionary hypothesis that mechanisms controlling food collection during the life cycle of solitary ancestors of honey bees have been coopted and remodeled to control foraging decisions in extant honey bees.

From this study it is evident that the ovary modulates sucrose perception, which in turn affects the volume of nectar collected. Bees with different numbers of ovarioles demonstrated different responses to sucrose concentration and this impacted on their foraging decisions regarding nectar loading. Extrapolating this into the human situation puts forward the possibility of ovarian regulation of sugar sensitivity and storage of calories. It is since long known that ovariectomized rats have higher body weight and increased appetite than their intact controls, a behavior that can be counteracted by estradiol treatment [1]. Similarly, postmenopausal women are more prone to gain weight than women before menopause [2]. Thus, from bees to women the ovaries are involved in keeping the metabolic balance.

\section{Concepts revised \\ Two papers on sex and maturational differences in human brain morphology}

\section{Pubertal Stage and Brain Anatomy in Girls}

Blanton RE, Cooney RE, Joormann J, Eugene F, Glover GH, Gotlib IH

Department of Psychiatry, Yale University, New Haven, CT, USA

Neuroscience 2012;217:105-112

Background: Studies of puberty have focused primarily on changes in hormones and on observable physical bodily characteristics. Little is known, however, about the nature of the relation between pubertal status and brain physiology and morphology. This is particularly important given findings that have linked the onset of puberty with both changes in cognitive functioning and increases in the incidence of depression and anxiety. 
Methods: The study examined relationships between pubertal stage, assessed by Tanner staging, and brain anatomy in a sample of 54 girls aged 9-15 years. Brain morphometric analysis was conducted using high-resolution magnetic resonance imaging (MRI). The hippocampus and amygdala were manually traced on MRI scans in all participants. Stepwise regression analyses were conducted with total intracranial volume (ICV), age, and pubertal status as the predictor variables and hippocampus and amygdala volumes as outcome variables.

Results: Pubertal status was significantly associated with left amygdala volume, after controlling for both age and intracranial volume (ICV). In addition, puberty was related to right hippocampus and amygdala volumes, after controlling for ICV. In contrast, no significant associations were found between age and hippocampal and amygdala volumes after controlling for pubertal status and ICV.

Conclusions: These findings highlight the importance of the relation between pubertal status and morphometry of the hippocampus and amygdala, and of limbic and subcortical structures that have been implicated in emotional and social behavior.

\section{Sex matters during adolescence: testosterone-related cortical thickness maturation differs between boys and girls}

Bramen JE, Hranilovich JA, Dahl RE, Chen J, Rosso C, Forbes EE, Dinov ID, Worthman CM, Sowell ER

Developmental Cognitive Neuroimaging Laboratory, Children's Hospital Los Angeles, Los Angeles, CA, USA

PloS One 2012;7:e33850

Background: Age-related changes in brain cortical thickness have been observed during adolescence, including thinning in frontal and parietal cortices, and thickening in the lateral temporal lobes. Studies have shown sex differences in hormone-related brain maturation when boys and girls are age-matched, however, because girls mature 1-2 years earlier than boys, these sex differences could be confounded by pubertal maturation.

Methods: To address puberty effects directly, this study assessed sex differences in testosterone-related cortical maturation by studying 85 boys and girls in a narrow age range and matched on sexual maturity. The authors expected that testosterone-by-sex interactions on cortical thickness would be observed in brain regions known from the animal literature to be high in androgen receptor (AR) expression.

Results: There were sex differences in associations between circulating testosterone and thickness in left inferior parietal lobule, middle temporal gyrus, calcarine sulcus, and right lingual gyrus, all regions known to be high in AR expression. Visual areas increased with testosterone in boys, but decreased in girls. All other regions were more impacted by testosterone levels in girls than boys.

Conclusions: The regional pattern of sex-by-testosterone interactions may have implications for understanding sex differences in behavior and adolescent-onset neuropsychiatric disorders.

These two papers add fuel to the longstanding discussion on gender differences in brain function and morphology. Some investigators still argue such differences are sparse or nonexistent but the present two papers clearly demonstrate such differences in addition to those related to the maturational stage. Although up-to-date methodology was employed for these noninvasive investigations, the techniques are still crude when compared with more invasive and less safe methods that may be used in animal experiments. Future more refined imaging techniques will allow more detailed studies and will most certainly take this field further in the not so far future. For the clinician the papers help to set references for morphometric analyses that may be of importance in investigations of patients with, e.g., pubertal and gender identity disorders. 


\section{Sexual dimorphism in the early life programming of serum leptin levels in European adolescents: the HELENA study}

Labayen I, Ruiz JR, Huybrechts I, Ortega FB, Rodriguez G, Dehenauw S, Breidenassel C, Jimenez-Pavon D, Vyncke KE, Censi L, Molnar D, Widhalm K, Kafatos A, Plada M, Diaz LE, Marcos A, Moreno LA, Gottrand F

Department of Nutrition and Food Science, University of the Basque Country, Vitoria, Spain

idoia.labayen@ehu.es

J Clin Endocrinol Metab 2011;96:E1330-1334

Background: Concentration of hormones, metabolites, and neurotransmitters during critical periods of early development has been suggested to preprogram brain development and metabolism later in life. Leptin is a hormone mainly produced and secreted into the circulation by the adipose tissue and plays a key role in the chronic control of energy balance and insulin sensitivity. In this study it was tested if a lower birth weight, as an indicator of adverse intrauterine environment, may be associated with higher serum leptin levels in European adolescents.

Methods: Fasting serum leptin was measured in 757 (429 females) European adolescents born at term aged $14.6 \pm 1.2$ years. Weight and height, was measured and body mass index was calculated. Birth weight, duration of pregnancy, and duration of breast-feeding were obtained from parental records. Duration of pregnancy and breast-feeding, pubertal status, center, body mass index, and physical activity were entered as confounders in the analyses.

Results: There was a significant interaction effect between sex and birth weight on serum leptin levels. Body weight at birth was negatively and significantly associated with serum leptin levels only in female adolescents. The association persisted after further controlling for physical activity.

Conclusions: These findings provide further evidence for a sex-specific programming effect of birth weight on serum leptin levels. The results could also contribute to explain the detrimental health effects associated with lower birth weight, such as long-term increased risk of developing obesity and type 2 diabetes.

\section{Postterm birth is associated with greater risk of obesity in adolescent males}

Beltrand J, Soboleva TK, Shorten PR, Derraik JG, Hofman P, Albertsson-Wikland K, Hochberg Z, Cutfield WS

Liggins Institute, University of Auckland, Auckland, New Zealand

J Pediatr 2012;160:769-773

Background: Individuals who were born preterm appear to be more prone to insulin resistance, and small size at birth is associated with a variety of adult diseases, including type 2 diabetes, ischemic heart disease, and obesity. Postterm birth has no known associated long-term sequelae, but it has been linked to greater perinatal mortality and morbidity. However, it is known that prolonged gestation may lead to a suboptimal fetal environment through inadequate nutrition or physiological stress, resulting in longterm postnatal alterations in body composition.

Methods: To test the hypothesis that postterm birth (at $>42$ completed weeks of gestation or $>293$ days from the first day of the last menstrual period) adversely affects longitudinal growth and weight gain throughout childhood a total of 525 children (including 17 boys and 20 girls born postterm) were followed from birth to the age of 16 years. Weight and height were recorded prospectively throughout childhood, and the respective velocities from birth to end of puberty were calculated using a mathematical model.

Results: At birth, postterm girls were slimmer than term girls. At 16 years of age, postterm boys were $11.8 \mathrm{~kg}$ heavier than term boys. The rate of obesity was $29 \%$ in postterm boys and $7 \%$ in term boys, and the combined rate of overweight and obesity was $47 \%$ in postterm boys and $13 \%$ in term boys. BMI was higher in postterm boys at already 3 years of age, with the difference increasing thereafter. BMI and growth were similar in postterm and term girls.

Conclusion: In this postterm birth cohort, boys, but not girls, demonstrated accelerated weight gain during childhood, leading to greater risk of obesity in adolescence.

There are many studies indicating that individuals exposed to metabolic and nutritional alterations in the fetal environment may have increased risk of developing obesity and type 2 diabetes in 
adulthood. A recent article in Nature Reviews Endocrinology [3] highlighted two studies which reported female-specific effects of fetal programming on later risk of developing obesity or type 2 diabetes mellitus. One of those studies was by Labayen et al., see above. The other study showed that high insulin levels at birth were associated with a slow rate of growth in the first year of life in girls, but not in boys [4]. Thus, the uterine environment reflected as both hormonal and nutritional status of the mother will impact on adult life and health of offspring - especially for the daughters. On the other hand, the current study by Beltrand et al. showed that postterm boys, but not girls, experienced increased weight gain during childhood and higher risk of obesity in adolescence. It is clear that the uterine environment and fetal programming is important for future health, but to what extent there is a sexual dimorphism varies between studies and the endpoints that are examined.

\section{Diet-induced paternal obesity in the absence of diabetes diminishes the reproductive health of two subsequent generations of mice}

Fullston T, Palmer NO, Owens JA, Mitchell M, Bakos HW, Lane M

Research Centre for Reproductive Health Discipline of Obstetrics and Gynaecology, Level 3 Medical School South, Robinson Institute, University of Adelaide, Adelaide, SA, Australia

Hum Reprod 2012;27:1391-1400

Background: Obesity and related conditions, notably subfertility, are increasingly prevalent. Paternal influences are known to affect the health of the offspring, but the impact of paternal obesity and subfertility on the reproductive health of subsequent generations is not known.

Methods: A high-fat diet (HFD) was used to induce obesity (but not diabetes) in male mice, which were subsequently mated to normal-weight females. First-generation offspring were raised on a control diet and their gametes were investigated for signs of subfertility. Second-generation offspring were generated from both first-generation sexes and their gametes were similarly assessed.

Results: HFD-induced paternal initiation of subfertility was found in both male and female offspring of two generations of mice. Furthermore, diminished reproductive and gamete functions were found to be transmitted through the first-generation paternal line to both sexes of the second generation and via the first-generation maternal line to second-generation males. Previous findings that male obesity alters the epigenome of sperm could provide a basis for the developmental programming of subfertility in subsequent generations.

Conclusions: This observation of paternal transmission of diminished reproductive health to future generations could have significant implications for the transgenerational amplification of subfertility observed worldwide in humans.

\section{Nature, nurture or nutrition? Impact of maternal nutrition on maternal care, offspring development and reproductive function}

Connor KL, Vickers MH, Beltrand J, Meaney MJ, Sloboda DM

Liggins Institute, University of Auckland, Grafton, Auckland, New Zealand

d.sloboda@auckland.ac.nz

J Physiol 2012;590:2167-2180

Background: Offspring of mothers fed a high-fat (HF) diet during pregnancy and lactation have been shown to enter puberty early and to be hyperleptinemic, hyperinsulinemic and obese as adults. Poor maternal care and bonding can also impact offspring development and disease risk. This study hypothesized that prenatal nutrition would affect maternal care and that an interaction may exist between a maternal HF diet and maternal care, subsequently impacting on offspring phenotype.

Methods: Wistar rats were mated and randomized to control dams fed a control diet (CON) or dams fed a HF diet from conception until the end of lactation (HF). Maternal care was assessed between postnatal day (P)3 and P8. Postweaning (P22), offspring were fed a control CON or HF diet. From P27, pubertal onset was assessed. At $\sim$ P105, estrous cyclicity was investigated.

Results: Maternal HF diet reduced maternal care; HF-fed mothers licked and groomed pups less than CON dams. Both female and male offspring of HF dams were lighter from birth to P11 than offspring 
of CON dams, but by P19 HF offspring were heavier than controls. Pups from HF-fed dams went into puberty early and this effect was exacerbated by a postweaning HF diet. Maternal and postweaning HF diets independently altered estrous cyclicity in females: female offspring of HF-fed mothers were more likely to have prolonged or persistent estrus, whilst female offspring fed a HF diet postweaning were more likely to have irregular estrous cycles and were more likely to have prolonged or persistent estrus.

Conclusion: Maternal HF nutrition during pregnancy and lactation results in a maternal obese phenotype and has a significant impact on maternal care during lactation. Maternal and postweaning nutritional signals, independent of maternal care, alter offspring body fat in prepuberty and female reproductive function in adulthood, which may be associated with advanced ovarian ageing and altered fertility.

In these animal studies it is shown that obese fathers affect the coming generations and give rise to subfertility among both male and female offspring. Obese mothers seem to affect reproductive function in daughters only, even if both male and female offspring went into puberty earlier and showed increased body weight after P19. These results indicate that the early nutritional environment can influence offspring development in a manner that alters reproductive potential and longterm health.

\section{Concepts revised and follow-up on a Yearbook 2009 (p. 79) paper}

\section{Oocyte formation by mitotically active germ cells purified from ovaries of reproductive-age women}

White YA, Woods DC, Takai Y, Ishihara O, Seki H, Tilly JL

Vincent Center for Reproductive Biology, Massachusetts General Hospital Vincent Department of Obstetrics and Gynecology, Massachusetts General Hospital, Boston, MA, USA

Nat Med 2012:18:413-421

Background: Germline stem cells that produce oocytes in vitro and fertilization-competent eggs in vivo have been identified in and isolated from adult mouse ovaries.

Methods: A fluorescence-activated cell sorting-based protocol that can be used with adult mouse ovaries and human ovarian cortical tissue to purify rare mitotically active cells that have a gene expression profile that is consistent with primitive germ cells was described and validated.

Results: Once established in vitro, these primitive germ cells could be expanded for months and spontaneously generated 35 - to $50-\mu \mathrm{m}$ oocytes, as determined by morphology, gene expression and haploid (1n) status. Injection of the human germline cells, engineered to stably express GFP, into human ovarian cortical biopsies lead to formation of follicles containing GFP-positive oocytes 1-2 weeks after xenotransplantation into immunodeficient female mice.

Conclusions: Ovaries of reproductive-age women, similar to adult mice, possess rare mitotically active germ cells that can be propagated in vitro as well as generate oocytes in vitro and in vivo.

In the 2009 issue of the Yearbook we reported on a finding that juvenile and adult mouse ovaries possess mitotically active germ cells, which completely objected to the current dogma that females of most mammalian species have lost their capacity for oocyte production at birth. A neonatal mouse female fetal stem cell line (FGSC) was established and cultured for more than 15 months. These FGSCs were infected with GFP virus and transplanted into ovaries of infertile mice. Transplanted cells underwent oogenesis and the mice produced offspring that had the GFP transgene. Thus, there is a possibility for oocyte renewal after birth! This year we can report that isolated egg-producing stem cells from the ovaries of reproductive age women were obtained and these cells were shown to produce what appear to be normal egg cells or oocytes. It seems to be possible also for human ovaries to produce new oocytes postnatally!

This controversial result was highlighted in several journals and web pages, e.g. Science Daily, please see http://www.sciencedaily.com/releases/2012/02/120226153641.htm and MDNEWS.com, please see 
http://www.mdnews.com/news/hd/2012_10/hd_662066. In Biology of Reproduction, Oatley and Hunt [5] describe what they consider to be the first evidence that oogonial stem cells can also be isolated from human ovaries, with similar technique as was used to obtain the mouse oogonial stem cells. The results from White's paper definitely challenge the old dogma of girls being born with their final number of oocytes.

\title{
Review \\ On female sexual behavior via neural PRs
}

\author{
Neural Progestin Receptors and Female Sexual Behavior \\ Mani SK, Blaustein JD \\ Department of Molecular \& Cellular Biology, Department of Neuroscience, Center on Addiction, Learning and \\ Memory, Baylor College of Medicine, Houston, TX, USA \\ Neuroendocrinology 2012 (E-pub ahead of print)
}

Background: The steroid hormone, progesterone, modulates neuroendocrine functions in the central nervous system resulting in integration of reproduction and reproductive behaviors in female mammals. Although it is widely recognized that progesterone's effects on female sex behavior are mediated by the classical neural progestin receptors (PRs) functioning as 'ligand-dependent' transcription factors to regulate genes and genomic networks, additional mechanisms of PR activation also contribute to the behavioral response. PRs can be activated in a ligand-independent manner by neurotransmitters, growth factors, cyclic nucleotides, progestin metabolites and mating stimuli. The rapid responses of progesterone may be mediated by a variety of PR types, including membrane-associated PRs or extranuclear PRs. Furthermore, these rapid, nonclassical progesterone actions involving cytoplasmic kinase signaling and/or extranuclear PRs also converge with the classical PR-mediated, transcription-dependent pathway to regulate reproductive behaviors.

Method and Results: This review summarizes some of the history of the study of the role of PRs in reproductive behaviors, and update the status of PR-mediated mechanisms involved in the facilitation of female sex behavior. An integrative model of PR activation via cross-talk and convergence of multiple signaling pathways is presented.

Conclusions: The original two-step classical model of PR activation has undergone substantial modifications and evolved into a highly complex integrative model involving multiple signaling pathways. Recent studies have provided insights into ligand-dependent and ligand-independent mechanisms of receptor activation and provided a blueprint for the integrative model for PR activation in the regulation of female sexual behavior. It is also becoming abundantly clear that multiple intra- and intercellular mechanisms share signaling components that potentially amplify and integrate signals from a variety of stimuli to achieve neuroendocrine integration required for complex processes like reproductive behaviors. Future studies will likely reveal further insights into the mechanisms by which the multiple signals converge and reinforce neuronal responses to environmental and behavioral events to alter steroid hormone effects on female reproductive behavior.

The classical nuclear progesterone receptor is present in two isoforms, A and B, which are transcribed from two different initiation sites of the same gene. Progesterone-facilitated lordosis (a behavior of crucial importance for female reproduction in rats) is completely eliminated in the PR-A null mutant mouse. PR-B null mutant mice showed a trend of suppression of P-facilitated sexual behavior. Taken together, the data suggest that PR-A is essential for progesterone-facilitated lordosis, and both isoforms are required for optimal facilitation by progesterone. Today the increasing number of reports on alternative signaling pathways, e.g. nonligand-activated receptors, membrane-bound receptors, neurotransmitters and growth factors, have amplified the complexity in progesterone responses and the pathways regulating sexual behavior. 
1. Wade GN: Gonadal hormones and behavioral regulation of body weight. Physiol Behav 1972;8:523-534.

2. Pasquali R, Casimirri F, Labate AM, Tortelli O, Pascal G, Anconetani B, et al: Body weight, fat distribution and the menopausal status in women. The VMH Collaborative Group. Int J Obes Relat Metab Disord 1994;18:614-621.

3. Wilson C: Reproductive endocrinology: sex-specific early life effects on metabolism. Nat Rev Endocrinol 2011;7:500.

4. Regnault N, Botton J, Heude B, Forhan A, Hankard R, Foliguet B, et al: Higher cord C-peptide concentrations are associated with slower growth rate in the first year of life in girls but not in boys. Diabetes 2011;60:2152-2159.

5. Oatley J, Hunt PA: Of mice and (wo)men: purified oogonial stem cells from mouse and human ovaries. Biol Reprod 2012;86:196. 